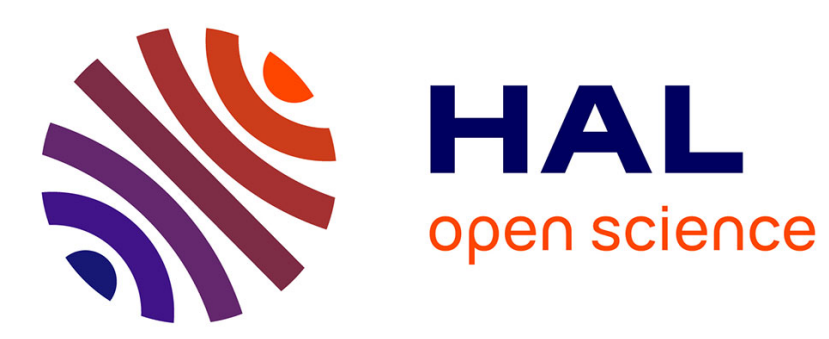

\title{
A Study of Waveguides with Sections of Proportional Sizes: Application to Wave Radiation
}

Denis Duhamel

\section{To cite this version:}

Denis Duhamel. A Study of Waveguides with Sections of Proportional Sizes: Application to Wave Radiation. Proceedings of the Eleventh International Conference on Computational Structures Technology, Sep 2012, Dubrovnik, Croatia. pp.1-18. hal-00782258

\section{HAL Id: hal-00782258 \\ https://hal.science/hal-00782258}

Submitted on 29 Jan 2013

HAL is a multi-disciplinary open access archive for the deposit and dissemination of scientific research documents, whether they are published or not. The documents may come from teaching and research institutions in France or abroad, or from public or private research centers.
L'archive ouverte pluridisciplinaire HAL, est destinée au dépôt et à la diffusion de documents scientifiques de niveau recherche, publiés ou non, émanant des établissements d'enseignement et de recherche français ou étrangers, des laboratoires publics ou privés. 


\title{
Paper ???
}

CCivil-Comp Press, 2012

Proceedings of the Eleventh International Conference

on Computational Structures Technology,

B.H.V. Topping, (Editor),

Civil-Comp Press, Stirlingshire, Scotland

\section{A Study of Waveguides with Sections of Proportional Sizes: Application to Wave Radiation}

\author{
D. Duhamel \\ Université Paris-Est, Laboratoire Navier \\ ENPC/LCPC/CNRS, Marne la Vallée, France
}

\begin{abstract}
Classically, waveguides are structures with uniform sections or are periodic along an axis. Vibrations of these waveguides can be described by wave modes and propagation constants relating the displacements and forces in two sections of the waveguide. Here, waveguides of non uniform sections are considered. More precisely, we consider waveguides which have sections with size increasing proportionally to the distance from an origin. This is for instance the case for a domain exterior to a convex body. Using a finite element model of a small section of the guide, the wave finite element method (WFE) can be used to find propagation constants and wave modes relating the displacements and forces at the two extremities of the finite element section. Then, it is shown that the wave modes and propagation constants in a section are simply related to the same quantities in other sections but for different frequencies. From this set of waves, general solutions in the waveguide can be computed. This approach allows an efficient solution by limiting the discretization to a very small part of the waveguide using only classical dynamic stiffness matrices, obtained by any finite element software. This approach can then be used for computing wave radiation in domains exterior to a convex body. The solution is projected onto these waves, allowing a clear separation between incoming and outgoing waves. Keeping only the outgoing part of the solution, wave radiation can be computed. It is shown that the approach is more efficient for high frequencies and can be complementary of the usual methods involving traditional finite or boundary elements.
\end{abstract}

Keywords: wave propagation, radiation, infinite domain, waveguide. 


\section{Introduction}

In this communication, we propose to study waveguides with sections of increasing sizes as in Figure 1. Classically waveguide structures are uniform or periodic along an axis, which is not the case here, so this paper describes how to adapt these classical methods to the present case.

One can find analytical or finite element models of uniform waveguides and people are generally interested by the computation of wave propagations and dispersion curves or by the determination of frequency response functions. For example, $[1,2]$ used a wave approach to study the vibrations of structural networks composed of sim-

ple uniform beams by analytical methods. The first numerical approaches were proposed by $[3,4]$ to approximate the cross-sectional deformations by finite elements. The authors of $[5,6]$ applied similar ideas to the calculation of wave propagations in rails using a finite element model of the cross-section of a rail. For general waveguides with a complex cross-section, the displacements in the cross-section can be described by the finite element method while the variation along the axis of symmetry is expressed as a wave function. Following these ideas, [7, 8, 9, 10, 11] developed the spectral finite element approach. This leads to efficient computations of dispersion relations and transfer functions but needs special matrices not given by usual FEM software.

More general waveguides can be studied by considering periodic structures. $\mathrm{Nu}-$ merous works provided interesting theoretical insights in the behavior of these structures, see for instance the work of [12] and the review paper by [13]. Mead also presented a general theory for wave propagation in periodic systems in $[14,15,16]$. He showed that the solution can be decomposed into an equal number of positive and negative-going waves. The approach is mainly based on Floquet's principle or the transfer matrix and the objective is to compute propagation constants relating the forces and displacements on the two sides of a single period and the waves associated to these constants. For complex structures FE models are used for the computation of the propagation constants and waves. The final objective is to compute dispersion relations to use them in energetic methods or to find transfer functions in the waveguide, see $[17,18,19,20]$. In [21] the general dynamic stiffness matrix for a periodic structure was found from the propagation constants and waves.

Here, a waveguide method, based on ideas related to wave finite elements as described in [17, 21, 22], is used to compute waveguides with homothetic cross-sections. Propagation constants and wave modes are introduced. It is shown how the solution in a domain of the waveguide can be computed for various frequencies using simple relations between domains of homothetic sizes and their propagating modes. The paper is divided into three sections and is outlined as follows. In section two, the principles of the method are described in general ways. In section three, examples are described to get a clear insight in the proposed approach before concluding remarks. 


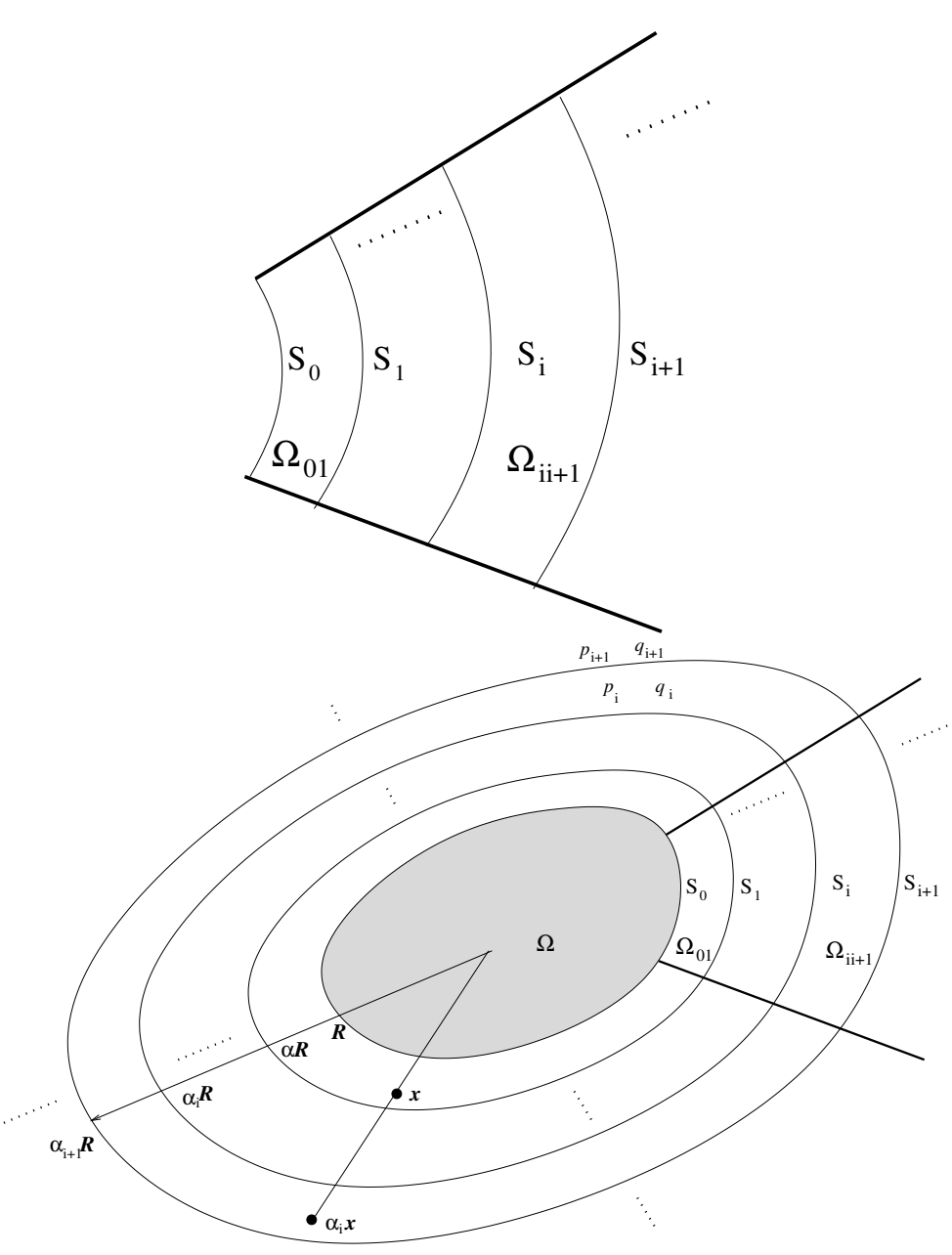

Figure 1: Two examples of waveguides : a simple waveguide and an exterior domain divided into zones of proportional sizes.

\section{Computation of waveguides}

\subsection{Behavior of a cell}

We consider waveguide structures as shown in Figure 1. A cell, defined as the domain $\Omega_{01}$ between the surfaces $S_{0}$ and $S_{1}$, is described by a finite element model. It is supposed that the outer surface $S_{1}$ has a size proportional to the inner surface $S_{0}$, which means that points on these surfaces are related by $\mathbf{x}_{1}=\alpha \mathbf{x}_{0}$ where $\alpha$ is a constant scale parameter. A cell can be meshed with an arbitrary number of elements using the full possibilities of usual finite element software. The discrete dynamic behavior of the cell obtained from a finite element model at a circular frequency $\omega$ is given by

$$
\left(\mathbf{K}-\omega^{2} \mathbf{M}\right) \mathbf{U}=\mathbf{F}
$$


where $\mathbf{K}$ and $\mathbf{M}$ are the stiffness and mass matrices respectively, $\mathbf{F}$ is the excitation vector and $\mathbf{U}$ the vector of the degrees of freedom. The stiffness and mass matrices could be obtained from any commercial finite element software. The sizes of these matrices depend on the number of elements used to mesh the layer and can be arbitrarily increased for a better precision of the results. Introducing the dynamic stiffness matrix of the layer $\mathbf{D}^{L}=\mathbf{K}-\omega^{2} \mathbf{M}$, decomposing into inner (i), outer (e) and interior (I) degrees of freedom, assuming that there are no external excitations on the interior nodes, and condensing the interior degrees of freedom lead to

$$
\left[\begin{array}{ll}
\mathbf{D}_{i i}^{L} & \mathbf{D}_{i e}^{L} \\
\mathbf{D}_{e i}^{L} & \mathbf{D}_{e e}^{L}
\end{array}\right]\left[\begin{array}{l}
\mathbf{U}_{i} \\
\mathbf{U}_{e}
\end{array}\right]=\left[\begin{array}{l}
\mathbf{F}_{i} \\
\mathbf{F}_{e}
\end{array}\right]
$$

The assumption that there are no excitations on the interior degrees of freedom is satisfied for free waves inside the structure for which the excitations on a layer are only produced from the adjacent layers. By symmetry of the stiffness and mass matrices, the dynamic stiffness matrix is also symmetric, which leads to ${ }^{t} \mathbf{D}_{i i}^{L}=\mathbf{D}_{i i}^{L},{ }^{t} \mathbf{D}_{e e}^{L}=\mathbf{D}_{e e}^{L}$ and ${ }^{t} \mathbf{D}_{i e}^{L}=\mathbf{D}_{e i}^{L}$, where the superscript $t$ means the transpose.

Instead of working with the current variables defined on surfaces $S_{0}$ and $S_{1}$, we will work with reference variables defined by

$$
\begin{aligned}
\mathbf{U}_{i} & =\mathbf{u}_{i} \\
\mathbf{U}_{e} & =\alpha^{-d} \mathbf{u}_{e} \\
\mathbf{F}_{i} & =\mathbf{f}_{i} \\
\mathbf{F}_{e} & =\alpha^{d} \mathbf{f}_{e}
\end{aligned}
$$

where $d=\left(s_{d}-1\right) / 2$ and $s_{d}$ is the space dimension. Relations (3) should be identical in terms of force densities and displacements. However, the nodal values of the excitation in the finite element model are obtained through an integration over surface elements and so already include an $\alpha^{2 d}$ contribution. The $\alpha^{2 d}$ term is the increase in size of surface $S_{1}$ compared to surface $S_{0}$. This leads finally to the $\alpha^{d}$ term in the relation involving discrete nodal values of the displacement. Thus, the behavior of a cell is now given by

$$
\left[\begin{array}{c}
\mathbf{f}_{i} \\
\mathbf{f}_{e}
\end{array}\right]=\left[\begin{array}{cc}
\mathbf{D}_{i i}^{L} & \alpha^{-d} \mathbf{D}_{i e}^{L} \\
\alpha^{-d} \mathbf{D}_{e i}^{L} & \alpha^{-2 d} \mathbf{D}_{e e}^{L}
\end{array}\right]\left[\begin{array}{l}
\mathbf{u}_{i} \\
\mathbf{u}_{e}
\end{array}\right]=\left[\begin{array}{cc}
\mathbf{D}_{i i} & \mathbf{D}_{i e} \\
\mathbf{D}_{e i} & \mathbf{D}_{e e}
\end{array}\right]\left[\begin{array}{l}
\mathbf{u}_{i} \\
\mathbf{u}_{e}
\end{array}\right]
$$

This matrix is also symmetric.

\subsection{Computation of waves}

For free wave propagation inside the waveguide, we look for solutions such that the relations on the boundaries can be written as

$$
\left\{\begin{aligned}
\mathbf{u}_{e} & =\lambda \mathbf{u}_{i} \\
\mathbf{f}_{e} & =-\lambda \mathbf{f}_{i}
\end{aligned}\right.
$$


Relations (4) and (5) yield

$$
\left(\mathbf{D}_{e i}+\lambda\left(\mathbf{D}_{i i}+\mathbf{D}_{e e}\right)+\lambda^{2} \mathbf{D}_{i e}\right) \mathbf{u}_{i}=0
$$

In this relation, the matrices $\mathbf{D}_{i i}$ and $\mathbf{D}_{e e}$ are symmetrical while $\mathbf{D}_{e i}$ is the transpose of $\mathbf{D}_{i e}$. As the transpose of the system in relation (6) leads to the system with $1 / \lambda$ instead of $\lambda$, one can see that if $\lambda$ is an eigenvalue, so $1 / \lambda$ is also an eigenvalue. We denote by $\left(\lambda_{i}, \Phi_{i}^{+}\right)$and $\left(1 / \lambda_{i}, \Phi_{i}^{-}\right)$the two sets of eigenvalues and eigenfunctions. The first set is associated with eigenvalues such that $\left|\lambda_{i}\right|<1$ or in case $\left|\lambda_{i}\right|=1$ with positive-going waves, meaning that energy propagates towards increasing values of the distance. The second set is associated with eigenvalues such that $\left|\lambda_{i}\right|>1$ or in case $\left|\lambda_{i}\right|=1$ with negative-going waves, meaning that energy propagates towards the origin.

We define the right and left eigenvectors by

$$
\begin{aligned}
& \mathbf{\Phi}_{j}=\left[\begin{array}{c}
\mathbf{u}\left(\lambda_{j}\right) \\
\left(\mathbf{D}_{i i}+\lambda_{j} \mathbf{D}_{i e}\right) \mathbf{u}\left(\lambda_{j}\right)
\end{array}\right] \\
& \boldsymbol{\Psi}_{l}=\left[\begin{array}{c}
\left(\mathbf{D}_{e e}+\lambda_{l} \mathbf{D}_{e i}\right) \mathbf{u}\left(\frac{1}{\lambda_{l}}\right) \\
\mathbf{u}\left(\frac{1}{\lambda_{l}}\right)
\end{array}\right]
\end{aligned}
$$

It can be checked that orthogonality conditions are given by

$$
S_{l j}=\Psi_{l} . \Phi_{j}=d_{l} \delta_{l j}
$$

and after normalization of the eigenvectors $\boldsymbol{\Phi}_{j}$, one can take $d_{l}=1$.

\subsection{Solutions in homothetic zones}

Consider now the situation of Figure 1, where the waveguide is made of an infinite number of layers of homothetic sizes such that the positions in zones $\Omega_{i i+1}$ and $\Omega_{i+1 i+2}$ are related by $\mathbf{x}_{i+1}=\alpha \mathbf{x}_{i}$, where $\alpha$ is a constant parameter. Using the results of the precedent section, eigenvalues and eigenvectors are computed in the domain $\Omega_{i i+1}$ between surfaces $S_{i}$ and $S_{i+1}$.

As neighboring cells have proportional sizes, it can be checked that the eigenvalue problem in the domain $\Omega_{i+1 i+2}$ is the same eigenvalue problem as for zone $\Omega_{i i+1}$ but for the frequency $\alpha \omega$. More precisely, one has an eigenvector in the domain $\Omega_{i+1 i+2}$ from an eigenvector in the domain $\Omega_{i i+1}$ by

$$
\begin{aligned}
\boldsymbol{\Phi}_{j}^{i+1}(\alpha \mathbf{x}, \omega) & =\boldsymbol{\Phi}_{j}^{i}(\mathbf{x}, \alpha \omega) \\
\lambda_{j}^{i+1}(\omega) & =\lambda_{j}^{i}(\alpha \omega)
\end{aligned}
$$

where $\boldsymbol{\Phi}_{j}^{i}(\mathbf{x}, \omega)$ denotes the eigenvectors (the value of the solution on the inner surface $S_{i}$ ) and $\lambda_{j}^{i}(\omega)$ the eigenvalues of zone $\Omega_{i i+1}$ at the circular frequency $\omega$. Finally, the eigenvectors and eigenvalues in any domain can be computed from those of the first domain by

$$
\begin{aligned}
\boldsymbol{\Phi}_{j}^{i}\left(\alpha^{i} \mathbf{x}, \omega\right) & =\boldsymbol{\Phi}_{j}^{0}\left(\mathbf{x}, \alpha^{i} \omega\right) \\
\lambda_{j}^{i}(\omega) & =\lambda_{j}^{0}\left(\alpha^{i} \omega\right)
\end{aligned}
$$


The solution of a problem in the exterior domain around $\Omega$ can be decomposed on these eigenvectors. Defining the state vector on the surface $S_{i}$ by $\mathbf{s}^{i}={ }^{t}\left(\mathbf{u}_{i}, \mathbf{f}_{i}\right)$, the decomposition of the solution in term of waves with amplitudes $\mathbf{a}^{i}$ is given by

$$
\mathbf{s}^{i}=\boldsymbol{\Phi}^{i} \cdot \mathbf{a}^{i}=\sum_{j=1}^{j=M} a_{j}^{i} \boldsymbol{\Phi}_{j}^{i}
$$

where $\Phi^{i}$ is the matrix made of the $\Phi_{j}^{i}$ and $\mathbf{a}^{i}$ the vector of the $a_{j}^{i}$. So, between two consecutive layers, one gets

$$
\begin{aligned}
\mathbf{s}^{i+1} & =\boldsymbol{\Phi}^{i} \cdot \boldsymbol{\Lambda}^{i} \cdot \mathbf{a}^{i} \\
& =\boldsymbol{\Phi}^{i} \cdot \boldsymbol{\Lambda}^{i} \cdot \mathbf{\Psi}^{i} \cdot \mathbf{s}^{i} \\
& =\mathbf{T}^{i} \cdot \mathbf{s}^{i}
\end{aligned}
$$

where the different matrices are defined by

$$
\begin{aligned}
\boldsymbol{\Phi}^{i} & =\left[\boldsymbol{\Phi}_{1}^{i}, \ldots, \boldsymbol{\Phi}_{M}^{i}\right] \\
\boldsymbol{\Psi}^{i} & =\left[\boldsymbol{\Psi}_{1}^{i}, \ldots, \boldsymbol{\Psi}_{M}^{i}\right] \\
\boldsymbol{\Lambda}^{i} & =\operatorname{diag}\left[\lambda_{1}^{i}, \ldots, \lambda_{M}^{i}\right] \\
\mathbf{T}^{i} & =\boldsymbol{\Phi}^{i} \cdot \boldsymbol{\Lambda}^{i} \cdot \boldsymbol{\Psi}^{i}
\end{aligned}
$$

and $M=2 N$ if we retain $N$ positive-going and $N$ negative-going waves. From the properties seen previously, one has

$$
\begin{aligned}
\boldsymbol{\Phi}^{i}(\omega) & =\boldsymbol{\Phi}^{i-1}(\alpha \omega)=\boldsymbol{\Phi}^{0}\left(\alpha^{i} \omega\right) \\
\boldsymbol{\Psi}^{i}(\omega) & =\boldsymbol{\Psi}^{i-1}(\alpha \omega)=\boldsymbol{\Psi}^{0}\left(\alpha^{i} \omega\right) \\
\boldsymbol{\Lambda}^{i}(\omega) & =\boldsymbol{\Lambda}^{i-1}(\alpha \omega)=\boldsymbol{\Lambda}^{0}\left(\alpha^{i} \omega\right) \\
\mathbf{T}^{i}(\omega) & =\mathbf{T}^{i-1}(\alpha \omega)=\mathbf{T}^{0}\left(\alpha^{i} \omega\right)
\end{aligned}
$$

So, for the complete exterior domain until surface $S_{n}$, one has

$$
\mathbf{s}^{n}=\mathbf{T}^{n-1} \ldots \mathbf{T}^{0} \mathbf{s}^{0}=\mathbf{T}^{0}\left(\alpha^{n-1} \omega\right) \mathbf{T}^{0}\left(\alpha^{n-2} \omega\right) \ldots \mathbf{T}^{0}(\omega) \mathbf{s}^{0}=\mathbf{T}^{\mathrm{tot}} \mathbf{s}^{0}
$$

\subsection{Application to radiation problems}

For the computation of wave radiations in the exterior domain of Figure 1, the boundary condition must be such that the amplitudes of the negative going waves are null, meaning that

$$
\Psi_{j}^{n}(\omega) \mathbf{T}^{\text {tot }} \mathbf{s}^{0}=0
$$

for all $j$ associated to incoming waves. The state vector on surface $S_{0}$ is written as

$$
\mathbf{s}^{0}=\left[\begin{array}{c}
\mathbf{u}_{0} \\
\mathbf{f}_{0}
\end{array}\right]
$$




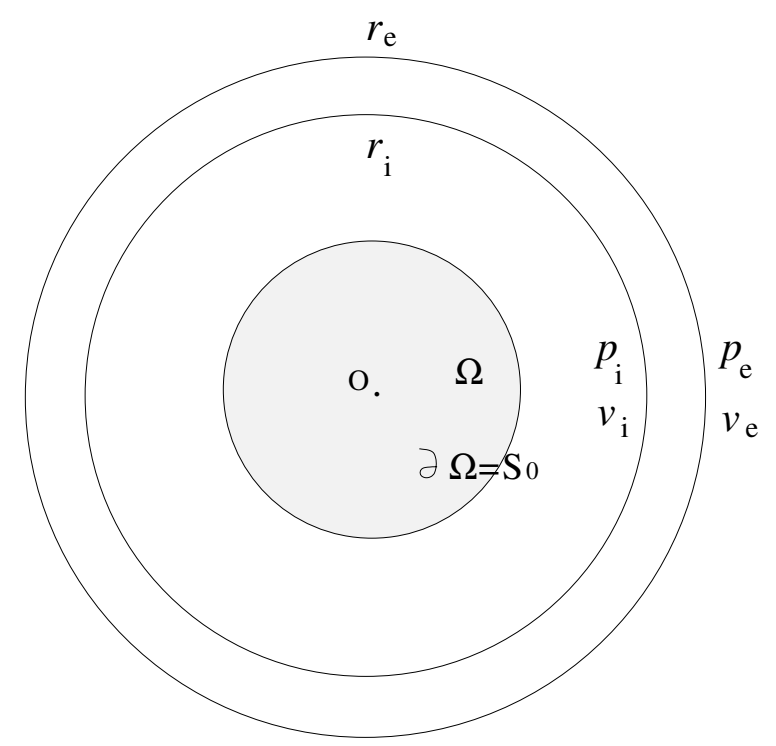

Figure 2: Annular domain.

and the matrix as

$$
\boldsymbol{\Psi}^{-}(\omega) \mathbf{T}^{\mathrm{tot}}=\left[\begin{array}{ll}
\mathbf{F}_{0} & \mathbf{Q}_{0}
\end{array}\right]
$$

where $\Psi^{-}(\omega)$ is a matrix made from the left vectors $\Psi_{j}^{n}$ associated to negative-going waves. Finally, the boundary condition can be written as the impedance condition

$$
\mathbf{F}_{0} \mathbf{u}_{0}+\mathbf{Q}_{0} \mathbf{f}_{0}=0
$$

This gives the relation on the surface of $\Omega$ approximating the radiation condition.

Note from relation (15) that it is possible to compute the total matrix for different frequencies by the following recurrence relation

$$
\mathbf{T}^{\mathrm{tot}}(\omega)=\mathbf{T}^{\mathrm{tot}}(\alpha \omega) \mathbf{T}^{0}(\omega)
$$

So beginning by the highest frequency, the different total matrices can be computed by determining only $\mathbf{T}^{0}(\omega)$ at each frequency and by computing the product of relation (20).

\section{Examples}

\subsection{Equations in an annular domain}

Consider the annular domain of figure 2 with the internal radius $r_{i}$ and the external radius $r_{e}$. This domain is exterior to the surface of a circular body $\Omega$. As an example, 
we are looking for the acoustic pressure $p$, solution of the eigenvalue problem

$$
\begin{aligned}
\Delta p+k^{2} p & =0 \\
\sqrt{r_{e}} p_{e} & =\lambda \sqrt{r_{i}} p_{i} \\
\frac{\sqrt{r_{e}}}{i k} \frac{\partial p_{e}}{\partial r} & =\lambda \frac{\sqrt{r_{i}}}{i k} \frac{\partial p_{i}}{\partial r}
\end{aligned}
$$

where $p_{i}, p_{e}$ are the internal and external values of the pressure $p$, the last line is the relation between the inner and outer velocities, $v_{i}$ and $v_{e}$. and $\lambda$ is the eigenvalue. The state vector is defined as

$$
\mathbf{s}(r)=\left[\begin{array}{c}
\sqrt{r} p(r, \theta) \\
\frac{\sqrt{r}}{i k} \frac{\partial p}{\partial r}(r, \theta)
\end{array}\right]
$$

and so is made of a first component proportional to the pressure and a second component proportional to the velocity.

The solution of the partial differential equation in the annular domain is given by

$$
p(r, \theta)=\sum_{n=-\infty}^{\infty}\left(a_{n} H_{n}^{1}(k r)+b_{n} H_{n}^{2}(k r)\right) e^{i n \theta}
$$

where $a_{n}, b_{n}$ are complex coefficients and $H_{n}^{1}$ and $H_{n}^{2}$ are Hankel functions of order $n$ of first and second types respectively.

The eigenvalue problem (21) can be written as

$$
\left[\begin{array}{ll}
\sqrt{r_{e}} H_{n}^{1}\left(k r_{e}\right) & \sqrt{r_{e}} H_{n}^{2}\left(k r_{e}\right) \\
\sqrt{r_{e}} \frac{\partial}{\partial r} H_{n}^{1}\left(k r_{e}\right) & \sqrt{r_{e}} \frac{\partial}{\partial r} H_{n}^{2}\left(k r_{e}\right)
\end{array}\right]\left[\begin{array}{l}
a_{n} \\
b_{n}
\end{array}\right]=\lambda\left[\begin{array}{ll}
\sqrt{r_{i}} H_{n}^{1}\left(k r_{i}\right) & \sqrt{r_{i}} H_{n}^{2}\left(k r_{i}\right) \\
\sqrt{r_{i}} \frac{\partial}{\partial r} H_{n}^{1}\left(k r_{i}\right) & \sqrt{r_{i}} \frac{\partial}{\partial r} H_{n}^{2}\left(k r_{i}\right)
\end{array}\right]\left[\begin{array}{l}
a_{n} \\
b_{n}
\end{array}\right]
$$

leading to

$$
\left|\begin{array}{ll}
\sqrt{r_{e}} H_{n}^{1}\left(k r_{e}\right)-\lambda \sqrt{r_{i}} H_{n}^{1}\left(k r_{i}\right) & \sqrt{r_{e}} H_{n}^{2}\left(k r_{e}\right)-\lambda \sqrt{r_{i}} H_{n}^{2}\left(k r_{i}\right) \\
\sqrt{r_{e}} \frac{\partial}{\partial r} H_{n}^{1}\left(k r_{e}\right)-\lambda \sqrt{r_{i}} \frac{\partial}{\partial r} H_{n}^{1}\left(k r_{i}\right) & \sqrt{r_{e}} \frac{\partial}{\partial r} H_{n}^{2}\left(k r_{e}\right)-\lambda \sqrt{r_{i}} \frac{\partial}{\partial r} H_{n}^{2}\left(k r_{i}\right)
\end{array}\right|=0
$$

The determinant is a quadratic function of $\lambda$. For the $\lambda^{2}$ term, using the following relation $H_{n}^{\prime}(z)=-H_{n+1}(z)+\frac{n}{z} H_{n}$ between Hankel functions, one gets

$$
\begin{aligned}
& k r_{i}\left(H_{n}^{1}\left(k r_{i}\right) H_{n}^{2^{\prime}}\left(k r_{i}\right)-H_{n}^{1^{\prime}}\left(k r_{i}\right) H_{n}^{2}\left(k r_{i}\right)\right) \\
= & -k r_{i}\left(H_{n}^{1}\left(k r_{i}\right) H_{n+1}^{2}\left(k r_{i}\right)-H_{n+1}^{1}\left(k r_{i}\right) H_{n}^{2}\left(k r_{i}\right)\right) \\
= & -\frac{4 i}{\pi}
\end{aligned}
$$

For the constant coefficient, the same computation also leads to $-\frac{4 i}{\pi}$

For the linear coefficient, one gets

$$
-k \sqrt{r_{i} r_{e}}\left(H_{n}^{1}\left(k r_{i}\right) H_{n}^{2^{\prime}}\left(k r_{e}\right)+H_{n}^{1}\left(k r_{e}\right) H_{n}^{2^{\prime}}\left(k r_{i}\right)-H_{n}^{2}\left(k r_{i}\right) H_{n}^{1^{\prime}}\left(k r_{e}\right)-H_{n}^{2}\left(k r_{e}\right) H_{n}^{1^{\prime}}\left(k r_{i}\right)\right)
$$


The precedent relations lead to the simplified equation

$$
\begin{aligned}
& \lambda^{2}+\frac{\pi k}{4 i} \sqrt{r_{i} r_{e}}\left(H_{n}^{1}\left(k r_{i}\right) H_{n}^{2^{\prime}}\left(k r_{e}\right)+H_{n}^{1}\left(k r_{e}\right) H_{n}^{2^{\prime}}\left(k r_{i}\right)\right. \\
& \left.-H_{n}^{2}\left(k r_{i}\right) H_{n}^{1^{\prime}}\left(k r_{e}\right)-H_{n}^{2}\left(k r_{e}\right) H_{n}^{1^{\prime}}\left(k r_{i}\right)\right) \lambda+1=0
\end{aligned}
$$

It can be seen that the eigenvalues come in inverse pairs $\lambda_{n}$ and $1 / \lambda_{n}$. Denoting by $\Phi_{n}^{+}$and $\Phi_{n}^{-}$the eigenvectors respectively associated to $\lambda_{n}$ and $1 / \lambda_{n}$, the solutions of equation (28) lead to the decomposition

$$
\mathbf{s}\left(r_{i}\right)=\left[\begin{array}{c}
\sqrt{r_{i}} p\left(r_{i}, \theta\right) \\
\frac{\sqrt{r_{i}}}{i k} \frac{\partial p}{\partial r}\left(r_{i}, \theta\right)
\end{array}\right]=\sum_{n=-\infty}^{\infty}\left(a_{n}^{+} \Phi_{n}^{+}\left(r_{i}\right)+a_{n}^{-} \Phi_{n}^{-}\left(r_{i}\right)\right) e^{i n \theta}
$$

with the eigenvectors given by

$$
\begin{aligned}
& \mathbf{\Phi}_{n}^{+}\left(r_{i}\right)=\left[\begin{array}{c}
\left\{-\left(\sqrt{r_{e}} H_{n}^{2}\left(k r_{e}\right)-\lambda_{n} \sqrt{r_{i}} H_{n}^{2}\left(k r_{i}\right)\right) \sqrt{r_{i}} H_{n}^{1}\left(k r_{i}\right)\right. \\
\left.+\left(\sqrt{r_{e}} H_{n}^{1}\left(k r_{e}\right)-\lambda_{n} \sqrt{r_{i}} H_{n}^{1}\left(k r_{i}\right)\right) \sqrt{r_{i}} H_{n}^{2}\left(k r_{i}\right)\right\} \\
\frac{1}{i k}\left\{-\left(\sqrt{r_{e}} H_{n}^{2}\left(k r_{e}\right)-\lambda_{n} \sqrt{r_{i}} H_{n}^{2}\left(k r_{i}\right)\right) \sqrt{r_{i}} \frac{\partial}{\partial r} H_{n}^{1}\left(k r_{i}\right)\right. \\
\left.+\left(\sqrt{r_{e}} H_{n}^{1}\left(k r_{e}\right)-\lambda_{n} \sqrt{r_{i}} H_{n}^{1}\left(k r_{i}\right)\right) \sqrt{r_{i}} \frac{\partial}{\partial r} H_{n}^{2}\left(k r_{i}\right)\right\}
\end{array}\right] \\
& =\left[\begin{array}{c}
2 i \sqrt{r_{i} r_{e}}\left(J_{n}\left(k r_{i}\right) Y_{n}\left(k r_{e}\right)-J_{n}\left(k r_{e}\right) Y_{n}\left(k r_{i}\right)\right) \\
\frac{1}{i k}\left(-\sqrt{r_{i} r_{e}} H_{n}^{2}\left(k r_{e}\right) \frac{\partial}{\partial r} H_{n}^{1}\left(k r_{i}\right)+\sqrt{r_{i} r_{e}} H_{n}^{1}\left(k r_{e}\right) \frac{\partial}{\partial r} H_{n}^{2}\left(k r_{i}\right)+\frac{4 i}{\pi} \lambda_{n}\right)
\end{array}\right]
\end{aligned}
$$

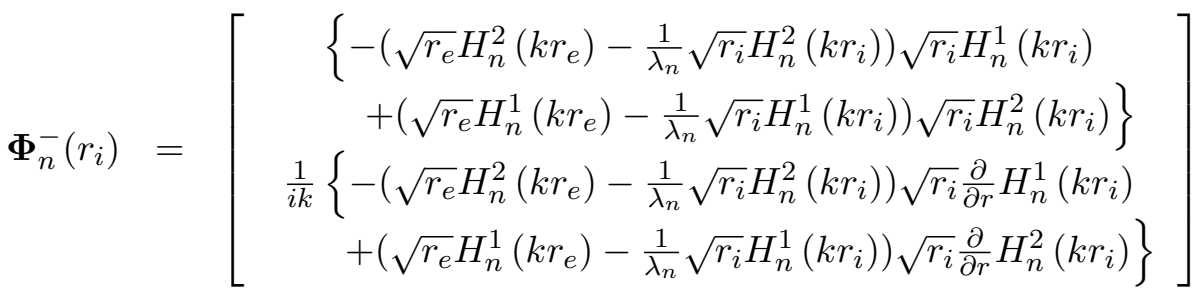

$$
\begin{aligned}
& =\left[\begin{array}{c}
2 i \sqrt{r_{i} r_{e}}\left(J_{n}\left(k r_{i}\right) Y_{n}\left(k r_{e}\right)-J_{n}\left(k r_{e}\right) Y_{n}\left(k r_{i}\right)\right) \\
\frac{1}{i k}\left(-\sqrt{r_{i} r_{e}} H_{n}^{2}\left(k r_{e}\right) \frac{\partial}{\partial r} H_{n}^{1}\left(k r_{i}\right)+\sqrt{r_{i} r_{e}} H_{n}^{1}\left(k r_{e}\right) \frac{\partial}{\partial r} H_{n}^{2}\left(k r_{i}\right)+\frac{4 i}{\pi \lambda_{n}}\right)
\end{array}\right]
\end{aligned}
$$

To clarify the precedent relations, consider the case for large values of $k r_{i}$ for which equation (28) simplifies to

$$
\lambda^{2}-2 \lambda \cos k\left(r_{e}-r_{i}\right)+1 \approx 0
$$

and the eigenvalues are approximately

$$
\begin{aligned}
\lambda_{n} & \approx e^{i k\left(r_{e}-r_{i}\right)} \\
1 / \lambda_{n} & \approx e^{-i k\left(r_{e}-r_{i}\right)}
\end{aligned}
$$

as if the curvature of the boundary was neglected. The development of relations (30) leads to the eigenvectors

$$
\begin{aligned}
\boldsymbol{\Phi}_{n}^{+}\left(r_{i}\right) \approx & {\left[\begin{array}{c}
\frac{4 i}{\pi k} \sin \left(k\left(r_{e}-r_{i}\right)\right) \\
-\frac{4}{\pi i k} \sin \left(k\left(r_{e}-r_{i}\right)\right)
\end{array}\right] } \\
\boldsymbol{\Phi}_{n}^{-}\left(r_{i}\right) \approx & {\left[\begin{array}{c}
\frac{4 i}{\pi k} \sin \left(k\left(r_{e}-r_{i}\right)\right) \\
\frac{4}{\pi i k} \sin \left(k\left(r_{e}-r_{i}\right)\right.
\end{array}\right] }
\end{aligned}
$$


These eigenvectors can be normalized to get

$$
\begin{aligned}
& \boldsymbol{\Phi}_{n}^{+}\left(r_{i}\right) \approx\left[\begin{array}{l}
1 \\
1
\end{array}\right] \\
& \boldsymbol{\Phi}_{n}^{-}\left(r_{i}\right) \approx\left[\begin{array}{c}
1 \\
-1
\end{array}\right]
\end{aligned}
$$

while

$$
\begin{aligned}
\boldsymbol{\Psi}_{n}^{+}\left(r_{i}\right) & \approx \frac{1}{2}\left[\begin{array}{ll}
1 & 1
\end{array}\right] \\
\boldsymbol{\Psi}_{n}^{-}\left(r_{i}\right) & \approx \frac{1}{2}\left[\begin{array}{ll}
1 & -1
\end{array}\right]
\end{aligned}
$$

One gets

$$
\boldsymbol{\Psi}^{0}\left(\alpha^{i} k\right) \boldsymbol{\Phi}^{0}\left(\alpha^{i-1} k\right)=\mathbf{I}
$$

and following relation (15), the total matrix for $N$ layers is

$$
\mathbf{T}^{\mathrm{tot}}=\left[\begin{array}{ll}
\boldsymbol{\Phi}^{(N-1)+} & \boldsymbol{\Phi}^{(N-1)-}
\end{array}\right]\left[\begin{array}{cc}
e^{i k\left(r_{N-1}-r_{0}\right)} & 0 \\
0 & e^{-i k\left(r_{N-1}-r_{0}\right)}
\end{array}\right]\left[\begin{array}{l}
\mathbf{\Psi}^{0+} \\
\boldsymbol{\Psi}^{0-}
\end{array}\right]
$$

and relation (18) yields

$$
\begin{aligned}
\boldsymbol{\Psi}^{N-} \mathbf{T}^{\mathrm{tot}} & =\boldsymbol{\Psi}^{N-}\left[\begin{array}{ll}
\boldsymbol{\Phi}^{(N-1)+} & \boldsymbol{\Phi}^{(N-1)-}
\end{array}\right]\left[\begin{array}{cc}
e^{i k\left(r_{N-1}-r_{0}\right)} & 0 \\
0 & e^{-i k\left(r_{N-1}-r_{0}\right)}
\end{array}\right]\left[\begin{array}{l}
\boldsymbol{\Psi}^{0+} \\
\boldsymbol{\Psi}^{0-}
\end{array}\right] \\
& =e^{-i k\left(r_{N-1}-r_{0}\right)} \mathbf{\Psi}^{0-}
\end{aligned}
$$

So the boundary condition is

$$
\Psi^{0-} \mathbf{s}^{0}=0
$$

or

$$
\sqrt{r} p-\frac{\sqrt{r}}{i k} \frac{\partial p}{\partial r}=0
$$

We get the usual relation

$$
\frac{\partial p}{\partial n}-i k p=0
$$

\subsection{Analytical results for the annular domain}

Some numerical values are computed, first for the pseudo wavenumber defined by $K=\frac{\log \lambda}{i\left(r_{e}-r_{i}\right)}$. A cylinder of radius $r_{i}=1 \mathrm{~m}$ is considered with a sound velocity $c=343 \mathrm{~m} / \mathrm{s}$. The first layer around the cylinder has a thickness of $0.01 \mathrm{~m}$ or $0.02 \mathrm{~m}$. Figures 3 and 4 present the real and imaginary parts of the wavenumber $K$ versus the order $n$ of the Hankel functions. It can be observed that for low orders the wavenumber is real and becomes with an imaginary part when a critical order is reached. The thickness of the layer has a limited influence on the wavenumber. On the contrary, 

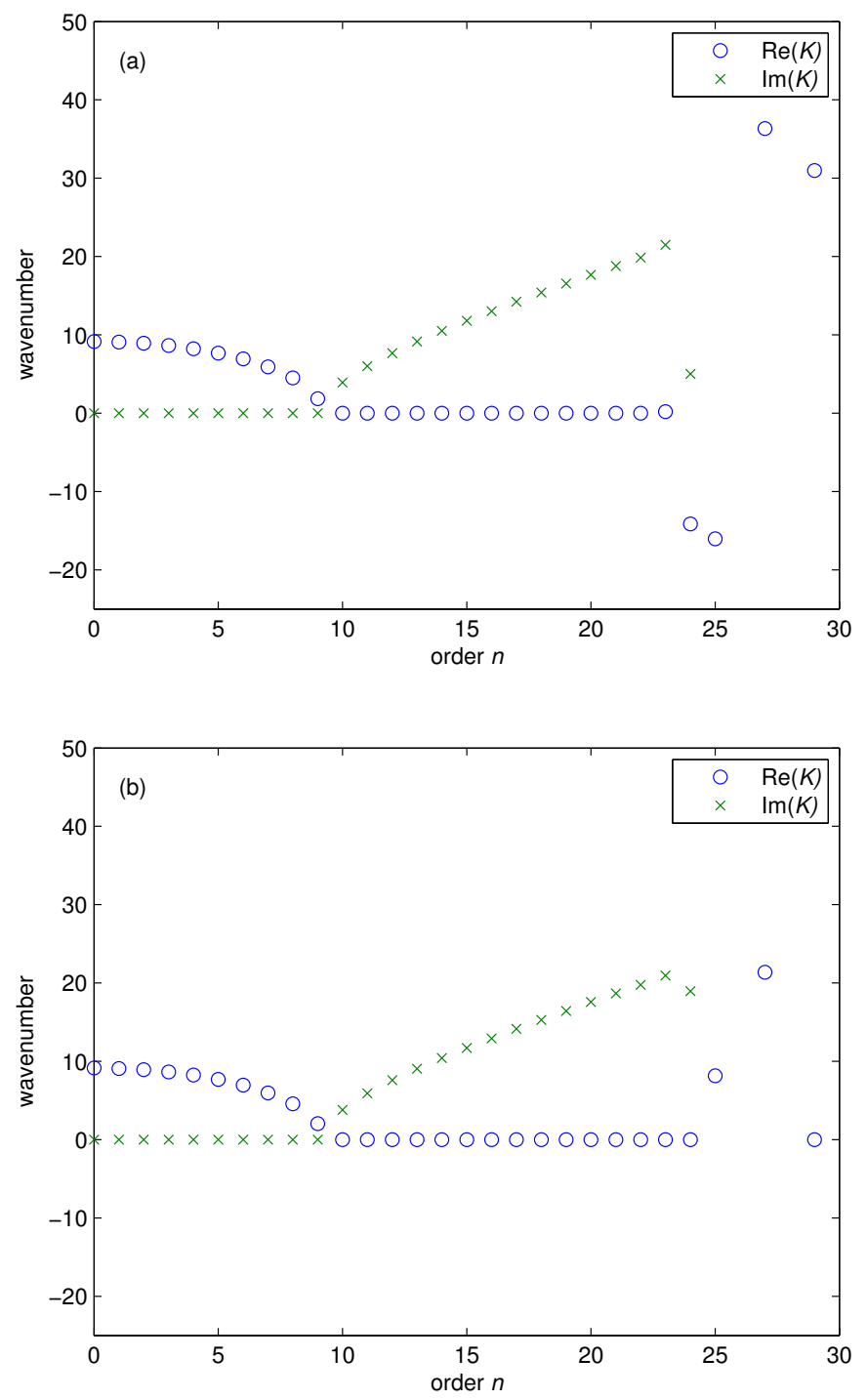

Figure 3: Wavenumbers for a thickness of $0.01 \mathrm{~m}$ (a) and $0.02 \mathrm{~m}$ (b) for the frequency $500 \mathrm{~Hz}$. 

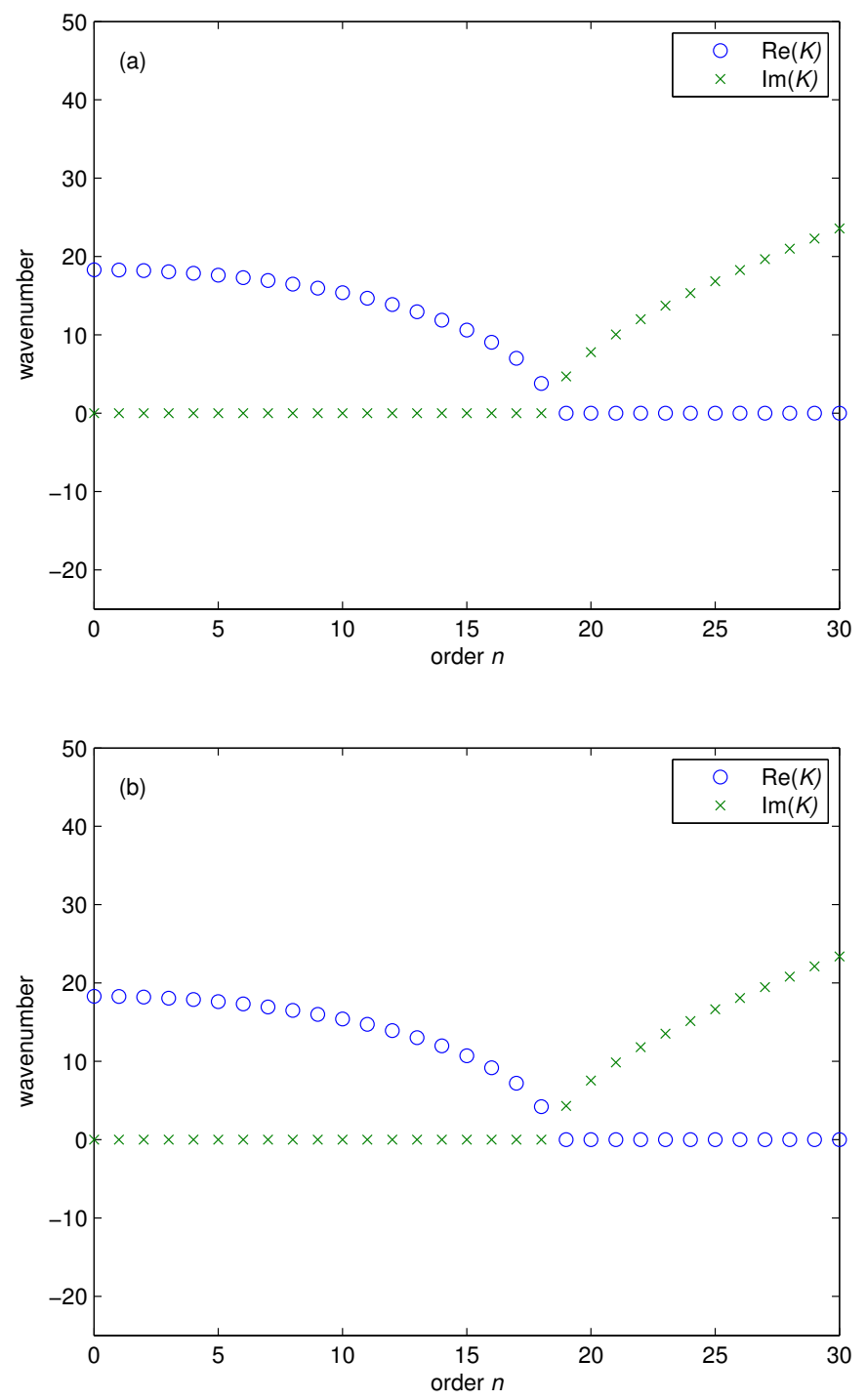

Figure 4: Wavenumbers for a thickness of $0.01 \mathrm{~m}$ (a) and $0.02 \mathrm{~m}$ (b) for the frequency $1000 \mathrm{~Hz}$. 


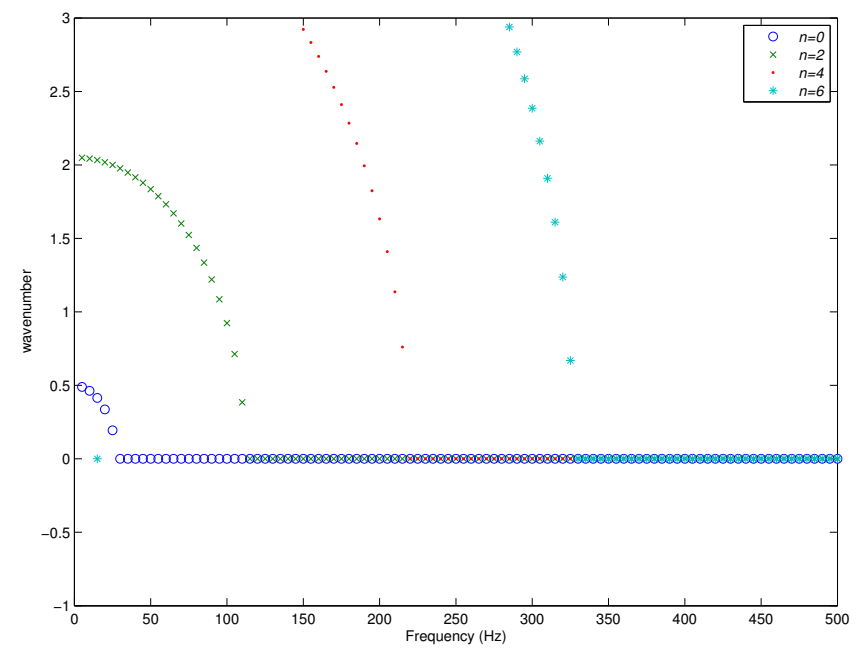

Figure 5: Imaginary part of the wavenumber for different orders $n$.

the comparison with Figure 4 shows that the frequency is very important. A higher frequency leads to more wavenumbers with purely real values. So it is analogous to classical waveguides with a cut-off frequency for which a given mode becomes propagative. Figure 5 presents the imaginary parts of the wavenumbers versus the frequency for different orders $n$. As long as the imaginary part of the wavenumber is null, the mode is propagating. It can be observed, for each mode, that the imaginary part becomes null when the frequency is higher than a critical frequency depending on the mode order. If we approximate the propagation in a layer by $p \approx e^{i(K r+n \theta)}$, the cut-off frequency is reached when the circumferential wavenumber $n / r_{i}$ equals the wavenumber of the problem $k$, that is when $\frac{n}{r_{i}}=k$ or in term of frequency $f=\frac{n c}{2 \pi r_{i}}$. This gives respectively $0 \mathrm{~Hz}, 109 \mathrm{~Hz}, 218 \mathrm{~Hz}$ and $328 \mathrm{~Hz}$ for the orders $n=0, n=2$, $n=4$ and $n=6$, which are good approximations of the cut-off frequencies seen in the figure.

\subsection{Surface impedance on a cylinder computed by WFE}

Consider the case of an annular domain meshed with one layer of four nodes linear acoustic elements. The cylinder is of radius $1 \mathrm{~m}$ and the sound velocity is $c=343 \mathrm{~m} / \mathrm{s}$. First, wavenumbers defined as $K=\frac{1}{i e} \log (\lambda)$ with $e$ the thickness of the layer are presented in figure 6 . The mesh contains 256 elements and the thickness is $e=0.001 \mathrm{~m}$. A very good agreement between numerical and analytical values is observed. Then the results in figure 7 present the error between the analytical impedance given by $-i \rho c \frac{H_{0}}{H_{1}}$ and the impedance found by the present method for the case of a uniform loading (independent of the direction). Three possible thicknesses are considered for the layer and the results are plotted for different numbers of elements in the mesh. 


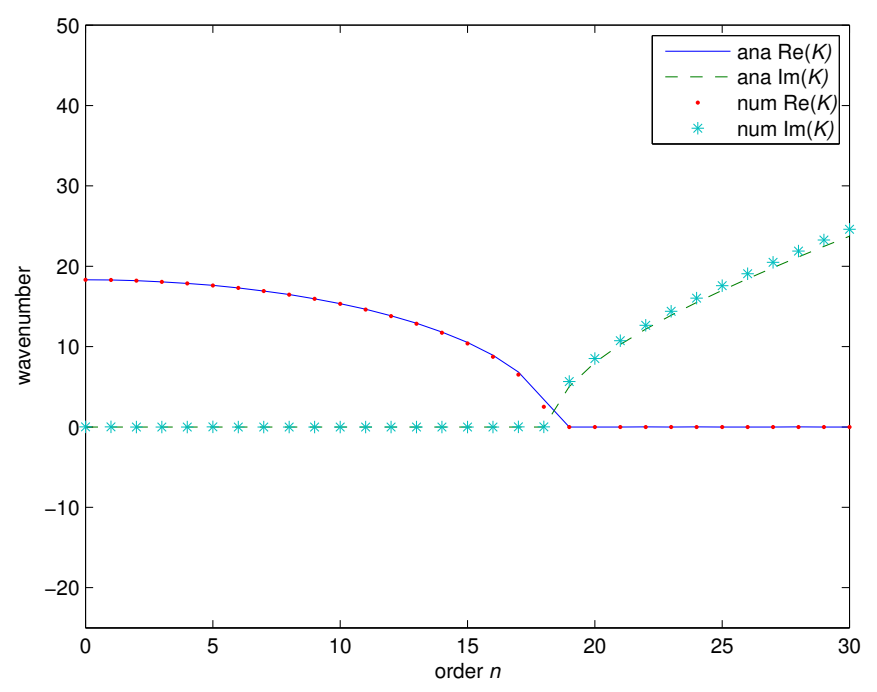

Figure 6: Comparison of analytical and numerical wavenumbers.

It can be seen that interesting results can be obtained with only one layer and with a limited number of degrees of freedom. The thickness $e=0.01 \mathrm{~m}$ is too large and lead to a loss of accuracy for high frequencies. So increasing the mesh density leads to much better results except when the thickness of the layer and the frequency are so large that the error is mainly controlled by a too large thickness.

In figure 8 , the error is plotted in the low frequency band for two thicknesses and different numbers of layers. It can be seen that increasing both the thickness and the number of layers can considerably increase the accuracy of the solution, while increasing only one parameter seems less efficient in this frequency range.

In figure 9 the same computation is done for three thicknesses of the first layer. The matrix is computed by the recurrence relation (20) starting from the highest frequency, here $2000 \mathrm{~Hz}$ and going towards lower frequencies by $f_{i+i}=f_{i} / \alpha$ with $f_{1}=2000 \mathrm{~Hz}$. A fixed number of 200 points in the frequency band was used. When $\alpha$ is small the accuracy is better for higher frequencies but a large number of frequency points is needed to get results for low frequencies. On the contrary for large thicknesses, the frequency range is larger at the expense of a reduced accuracy.

\section{Conclusion}

It has been shown that simple computations of eigenvalues and eigenvectors defined on a small rib around a radiating body for various frequencies could solve the problem of wave radiation in the complete exterior domain for any frequency as long as a sufficient number of layers or frequencies are involved in the computation. Tests on circular cases have provided accurate results. 

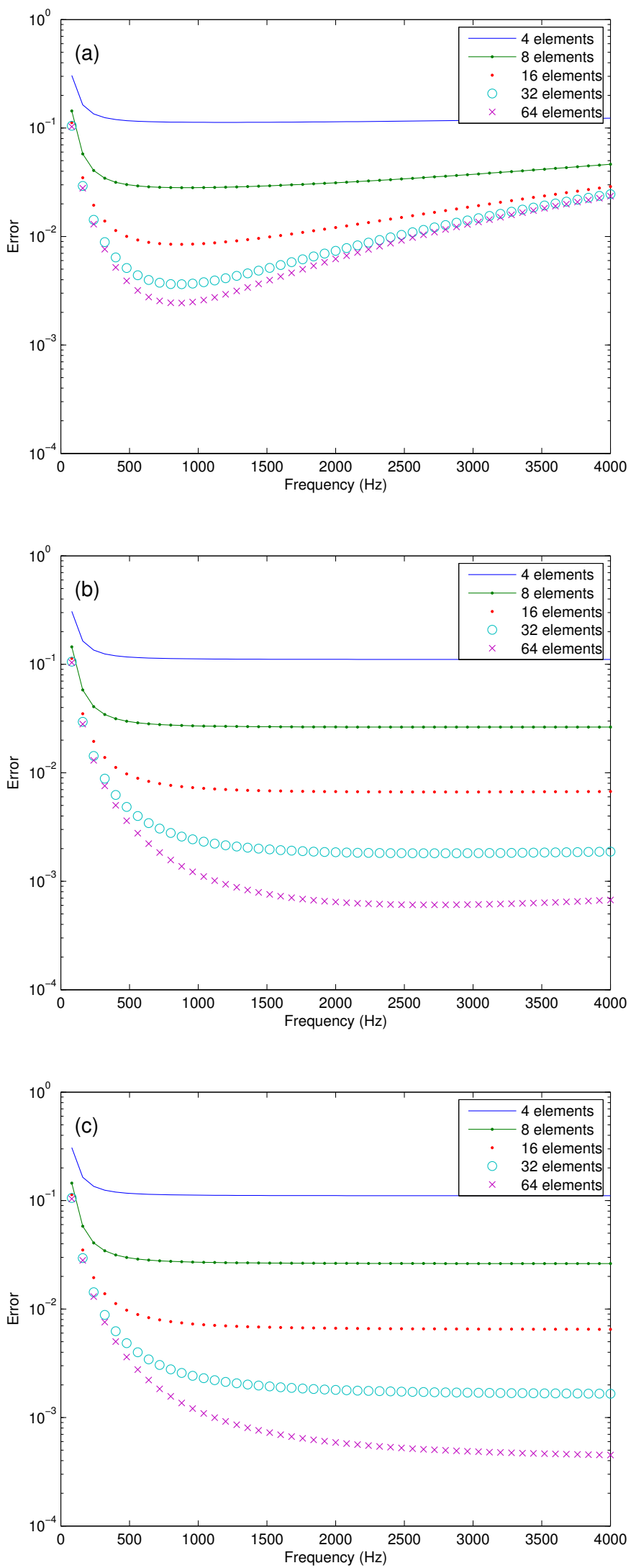

Figure 7: One layer with $e=0.01 \mathrm{~m}$ (a), with $e=0.001 \mathrm{~m}$ (b) and $e=0.0001 \mathrm{~m}$ (c). 

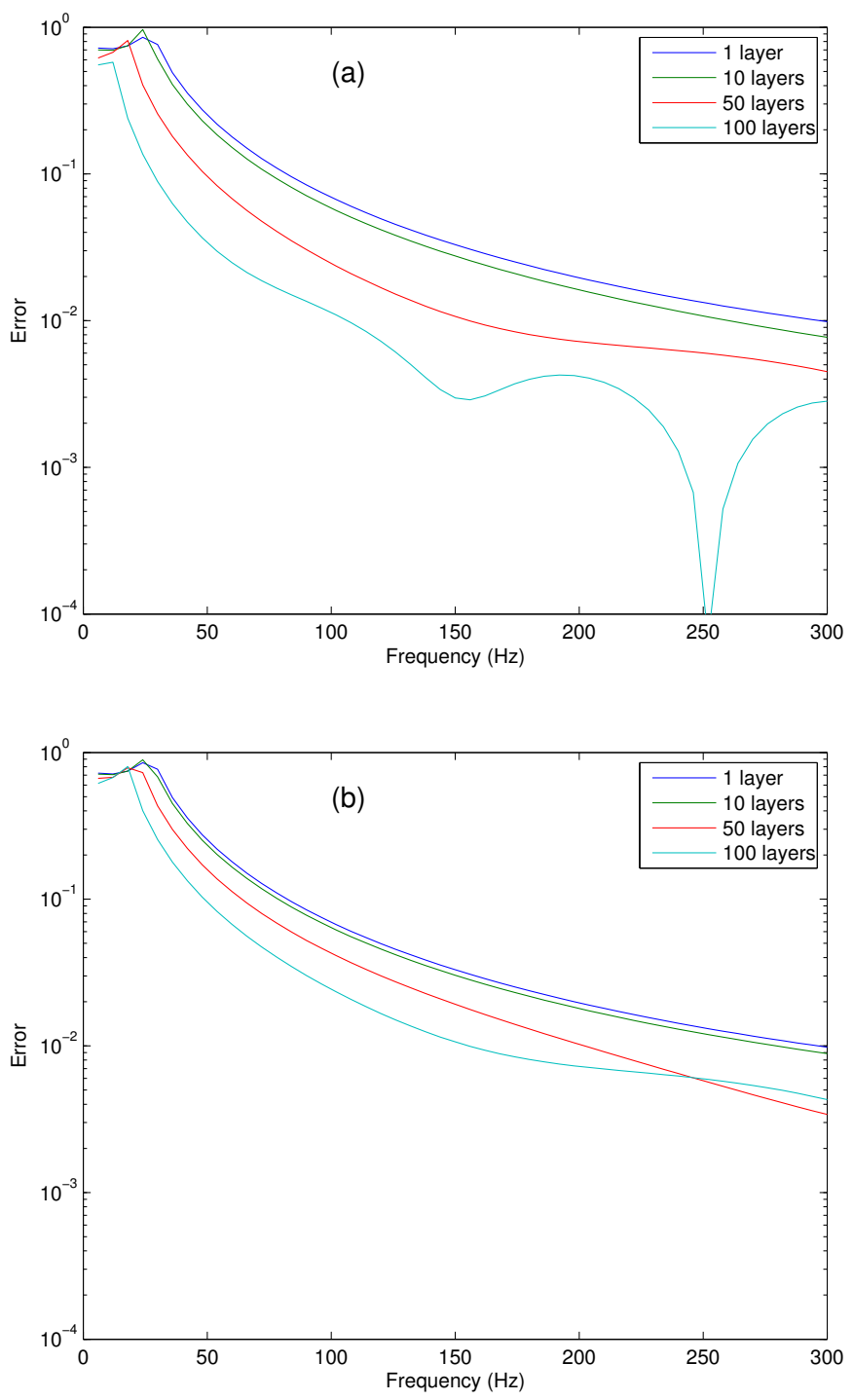

Figure 8: Errors for two thicknesses of the layer: $e=0.01 \mathrm{~m}$ (a) and $e=0.005 \mathrm{~m}$ (b). 


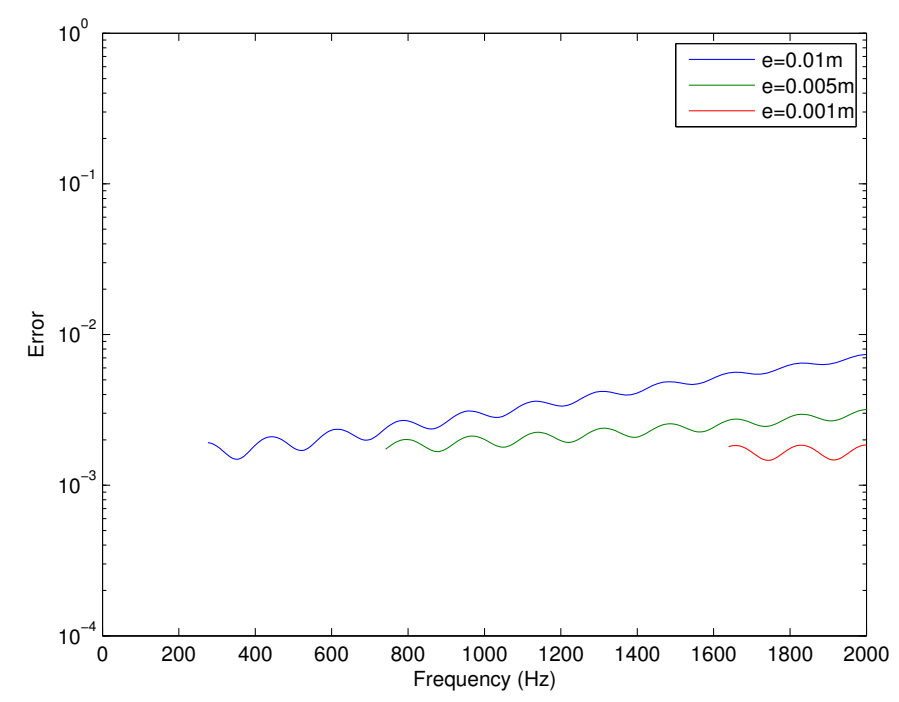

Figure 9: Error when using the recurrence relation.

\section{References}

[1] H. von Flotow, Disturbance propagation in structural networks, Journal of Sound and Vibration, 106 (1986) 433-450.

[2] L.S. Beale and M.L. Accorsi, Power flow in two- and three-dimensional frame structures, Journal of Sound and Vibration, 185 (1995) 685-702.

[3] S.B. Dong and R.B. Nelson, On natural vibrations and waves in laminated orthotropic plates, Journal of Applied Mechanics, 39 (3) (1972) 739-745.

[4] B. Aalami, Waves in prismatic guides of arbitrary cross section, Journal of Applied Mechanics, 40 (1973) 1067-1072.

[5] L. Gavric, Computation of propagating waves in free rail using a finite element technique, Journal of Sound and Vibration, 185 (3) (1995) 531-543.

[6] L. Gry, Dynamic modelling of railway track based on wave propagation, Journal of Sound and Vibration, 195 (1996) 477-505.

[7] S. Finnveden, Finite element techniques for the evaluation of energy flow parameters, Proc. Novem, Lyon (keynote paper), (2000).

[8] S. Finnveden, Evaluation of modal density and group velocity by a finite element method, Journal of Sound and Vibration, 273 (2004) 51-75.

[9] F. Birgersson, S. Finnveden and C.M. Nilsson, A spectral super element for modelling of plate vibration. Part 1: general theory, Journal of Sound and Vibration, 287 (2005) 297-314.

[10] I. Bartoli, A. Marzani, F. Lanza di Scalea and E. Viola, Modeling wave propagation in damped waveguides of arbitrary cross-section. Journal of Sound and Vibration, 295 (3-5) (2006) 685-707.

[11] F. Treyssède, Elastic waves in helical waveguides, Wave Motion, 45 (4) (2008) 
457-470.

[12] L. Brillouin, Wave propagation in periodic structures, New York: Dover, (1953).

[13] D.J. Mead, Wave propagation in continuous periodic structures: research contributions from Southampton, 1964-1995, Journal of Sound and Vibration, 190 (1996) 495-524.

[14] D.J. Mead, A general theory of harmonic wave propagation in linear periodic systems with multiple coupling, Journal of Sound and Vibration, 27 (1973) 235260.

[15] D.J. Mead, Wave propagation and natural modes in periodic systems: I. Monocoupled systems, Journal of Sound and Vibration, 40 (1975) 1-18.

[16] D.J. Mead, Wave propagation and natural modes in periodic systems: II. Multicoupled systems, with and without damping, Journal of Sound and Vibration, 40 (1975) 19-39.

[17] B.R. Mace, D. Duhamel, M.J. Brennan and L. Hinke, Finite element prediction of wave motion in structural waveguides, Journal of the Acoustical Society of America, 117 (2005) 2835-2843.

[18] J.M. Mencik and M.N. Ichchou, Multi-mode propagation and diffusion in structures through finite elements, European Journal of Mechanics A/Solids, 24 (2005) 877-898.

[19] L. Houillon, M.N. Ichchouh and L. Jezequel, Wave motion in thin-walled structures, Journal of Sound and Vibration, 281 (2005) 483-507.

[20] Y. Waki, B.R. Mace, M.J. Brennan, Numerical issues concerning the wave and finite element method for free and forced vibrations of waveguides, Journal of Sound and Vibration, 327 (2009) 92-108.

[21] D. Duhamel, B.R. Mace, M.J. Brennan, Finite element analysis of the vibrations of waveguides and periodic structures, Journal of Sound and Vibration, 294 (2006) 205-220.

[22] D. Duhamel, T.-M. Nguyen, Finite element computation of absorbing boundary conditions for time-harmonic wave problems, Computer Methods in Applied Mechanics and Engineering, 198 (2009) 3006-3019. 